Herzschr Elektrophys 2013 · 24:1-3

DOI 10.1007/s00399-013-0290-6

Online publiziert: 14. September 2013

(c) Springer-Verlag Berlin Heidelberg 2013

\section{Carsten W. Israel}

Klinik für Innere Medizin - Kardiologie, Diabetologie \& Nephrologie,

Evangelisches Krankenhaus Bielefeld, Bielefeld, Deutschland

\title{
Jahrestagung der Arbeitsgruppe Rhythmologie 2013
}

Die 1. Jahrestagung der Arbeitsgruppen „Herzschrittmacher“ und „Arrhythmie“ der Deutschen Gesellschaft für Kardiologie (DGK) fand 1989 unter Leitung von Herrn von Leitner, Hannover, statt und etablierte sich sofort als ein jährliches Treffen, das meist in der letzten Januarwoche stattfand (• Tab. 1). Dieses Meeting hatte von Beginn an einen besonderen Charakter, da es zum einen interdisziplinär war und nicht nur Kardiologen, sondern auch Herzchirurgen und Kardiotechniker aktiv teilnahmen. Zum anderen ist der Kreis der Personen, die sich so sehr für Arrhythmien interessieren, dass sie dafür durch die Bundesrepublik reisen und 2 Tage lang über Arrhythmien diskutieren, begrenzt. Damit kam sehr schnell ein „Familiengefühl“ auf, das bis heute anhält und eine Stärke der Arbeitsgruppe darstellt. Dieses Zusammengehörigkeitsgefühl war in Deutschland immer besonders präsent, da wir im Gegensatz zu anderen europäischen Ländern nur eine einzige nationale kardiologische Gesellschaft haben und die Tagung von den Arbeitsgruppen für bradykarde und tachykarde Arrhythmien gemeinsam durchgeführt wurde.

Bis 2003 fand die deutsche rhythmologische Jahrestagung als alleinstehender Kongress Ende Januar statt, zuletzt in Leipzig. Um die Kongressaktivitäten zu bündeln, wurde beschlossen, die Rhythmologie-Tagung zusammen mit der Herbsttagung am gleichen Ort zur gleichen Zeit stattfinden zu lassen, zum ersten Mal in Hamburg im Oktober 2004. Die Organisation beider Veranstaltungen fand weiter getrennt statt, die Abstracts beider Tagungen wurden separat in der Zeitschrift für Kardiologie veröffentlicht.
Die Zusammenlegung der Arbeitsgruppen „Herzschrittmacher“ und „Arrhythmien“ im Jahr 2007 war sinnvoll und logisch, da diese Bereiche inhaltlich in den letzten Jahren zusammengewachsen sind und viele deutsche Elektrophysiologen sowohl im Bereich Schrittmacher- und ICD-Implantation sowie -Nachsorge, aber auch bei der Behandlung von Vorhofflimmern und anderen Arrhythmien aktiv sind. Dennoch hat der gefühlte Verlust der alleinstehenden Tagung und der eigenständigen Arbeits- gruppe die Sorge ausgelöst, dass die Schrittmachertherapie auf Dauer nicht mehr mit der gleichen Sorgfalt durchgeführt würde, weil Foren und Unterstützung nicht mehr im selben Umfang zur Verfügung stehen.

Meiner Meinung nach ist es von großer Bedeutung, durch die Aktivitäten unserer Arbeitsgruppe Rhythmologie, durch die Rhythmologie-Jahrestagung und das offizielle Organ der Arbeitsgruppe „Herzschrittmachertherapie \& Elektrophysiologie“ die Qualität der Elektrophysiolo-

Tab. 1 Jahrestagungen und Tagungspräsidenten der Arbeitsgruppe 1 - Rhythmologie der DGK (früher Arbeitsgruppen „Herzschrittmacher" und "Arrhythmien")

\begin{tabular}{ll}
\hline Jahr & Tagungspräsident(en) \\
\hline 1989 & Prof. Dr. von Leitner, Hannover \\
\hline 1990 & Prof. Dr. P. Satter, Frankfurt \\
\hline 1991 & Prof. Dr. H. H. Hilger, Köln, und PD Dr. U. J. Winter, Essen \\
\hline 1992 & Prof. Dr. J. Meyer und Prof. Dr. N. Treese, Mainz \\
\hline 1993 & PD B. Lemke, Bochum \\
\hline 1994 & Prof. Dr. von Knorre, Rostock \\
\hline 1995 & Prof. Dr. G. Baumann und PD Dr. K. Stangl, Berlin \\
\hline 1996 & Prof. Dr. P. Hanrath, Aachen und PD Dr. M. Sigmund, Wiesbaden \\
\hline 1997 & Prof. Dr. V. Hombach und Dr. L. Binner, Ulm \\
\hline 1998 & Prof. Dr. H. U. Klein und Dr. W. M. Hartung, Magdeburg \\
\hline 1999 & Prof. Dr. E. Alt, München, zusammen mit dem Schrittmacher-Weltkongress in Berlin \\
\hline 2000 & Prof. Dr. H. Schieffer, Homburg und PD Dr. G. Fröhlig, Homburg \\
\hline 2001 & Prof. Dr. E. Gams und PD Dr. J. Winter, Düsseldorf \\
\hline 2002 & Prof. Dr. W. Hemmer, Stuttgart \\
\hline 2003 & PD Dr. H. Kottkamp, Leipzig und PD Dr. G. Hindricks, Leipzig \\
\hline 2004 & Prof. Dr. D. Pfeiffer, Leipzig \\
\hline 2005 & Prof. Dr. R. Strasser, Dresden \\
\hline 2006 & Prof. Dr. J. Neuzner, Kassel \\
\hline 2007 & Prof. Dr. W. Schöls, Duisburg \\
\hline 2008 & Prof. Dr. U. Wiegand, Lübeck \\
\hline 2009 & Prof. Dr. T. Lewalter, Paderborn \\
\hline 2010 & Prof. Dr. A. Schuchert, Neumünster \\
\hline 2011 & Prof. Dr. J. C. Geller, Bad Berka \\
\hline 2012 & Prof. Dr. N. Doll, Stuttgart \\
\hline 2013 & PD Dr. C. W. Israel, Bielefeld \\
\hline & \\
\hline
\end{tabular}




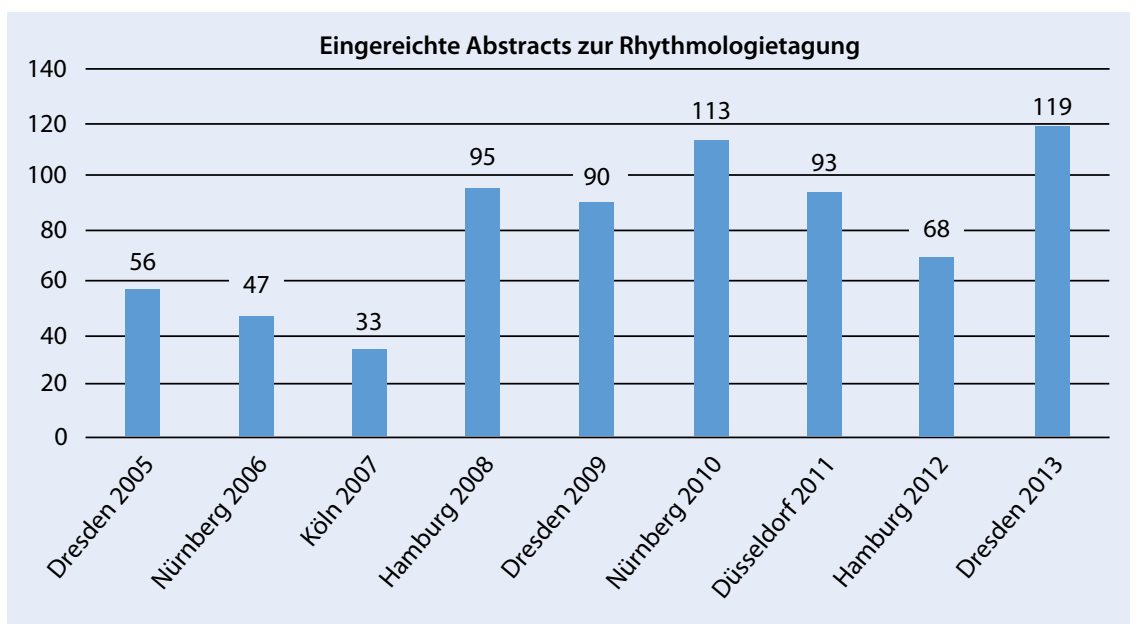

Abb. $1 \Delta$ Anzahl der angenommenen Abstracts der Rhythmologie-Jahrestagungen 2005 bis 2013

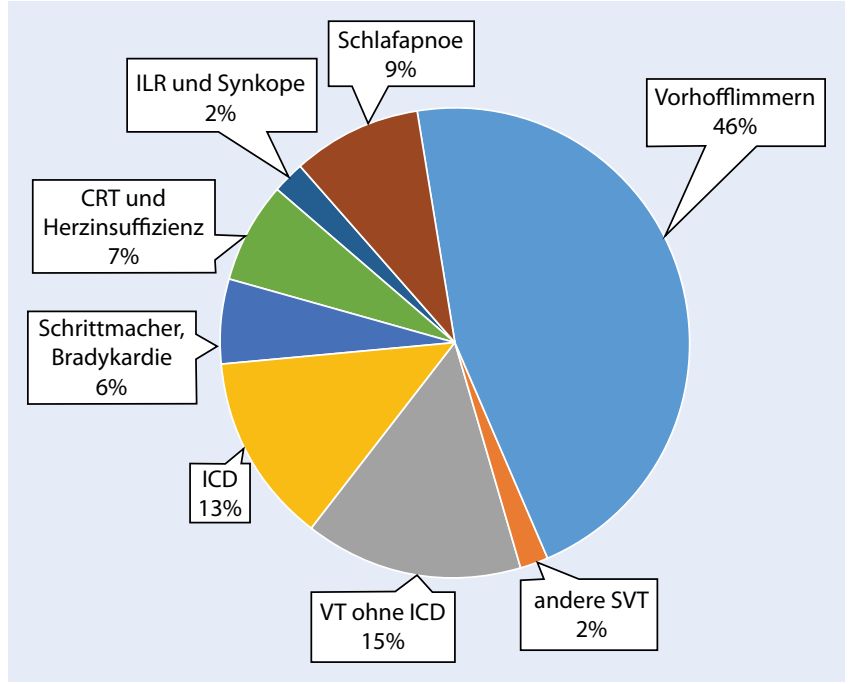

Abb. $2 \varangle$ Themen der Abstracts der Rhythmologie-Tagung 2013. CRT kardiale Resynchronisationstherapie, ICD implantierbarer Cardioverter/Defibrillator, ILR implantierbarer Loop-Rekorder, SVT supraventrikuläre Tachykardien, $V T$ ventrikuläre Tachykardien

gie in Deutschland in allen Bereichen, invasiv und nichtinvasiv, „Brady“ und „Tachy“, aufrechtzuerhalten bzw. zu verbessern und die praktische und theoretische Ausbildung sowie wissenschaftliche Entwicklung neuer Rhythmologen zu fördern. In dieser Hinsicht ist in den letzten Jahren viel geleistet worden; zu erwähnen sind hier etwa die Sachkundekurse Herzschrittmacher-, ICD-, CRT-Therapie und Elektrophysiologie oder die Erarbeitung eines Curriculums zur Erlangung der Zusatzqualifikation „Spezielle Rhythmologie", ebenso ein Fellowship-Programm in Zusammenarbeit mit der European Heart Rhythm Association (EHRA) und Sponsoren aus der Industrie.

Aber Sachkundekurse und die praktische Ausbildung in der Klinik können den Austausch von Wissen und Er- eigene Praxis akzeptieren. Die Wahrnehmung der wissenschaftlichen, aber auch organisatorischen und berufspolitischen Themen, die etwa während der Mitgliederversammlung auf der RhythmologieJahrestagung besprochen werden, sind für die eigene Arbeit von hoher Bedeutung und es sollte jedem Elektrophysiologen in Deutschland die Möglichkeit gegeben werden, hier aktiv teilzunehmen. Je besser wir uns als Arbeitsgruppe Rhythmologie organisieren und engagieren, desto mehr können wir für unser Fach erreichen. Allein die Wahrnehmung der mehr als 500 Mitglieder unserer Arbeitsgruppe mag dem ein oder anderen Elektrophysiologen klar machen, dass er nicht allein ist mit seinen Gedanken zu Mechanismen von Arrhythmien und deren Behandlung. Schließlich ist die Kommunikation mit Kollegen, die im gleichen Bereich tätig sind, fachlich und menschlich von großer Bedeutung für die eigene Entwicklung und die Akquise neuer Methoden und Therapien. Daher ist es außerordentlich wichtig, dass hierzu in Form der Jahrestagung der AG Rhythmologie der DGK ein nationales Forum existiert.

Vor diesem Hintergrund war die Verlegung der Rhythmologie-Jahrestagung auf einen Termin und Ort mit der Herbsttagung der DGK problematisch: Die Herbsttagung versteht sich in erster Linie als Fortbildungsveranstaltung, Präsentationen wissenschaftlicher Beiträge haben bei der Frühjahrstagung in Mannheim weitaus größeren Raum. Dies gilt nicht für die Rhythmologie-Jahrestagung: Viele eingereichte Abstracts sind für ein allgemein-kardiologisches Publikum in Mannheim zu speziell oder erscheinen in ihrer Bedeutung zu klein, um dort präsentiert werden zu können. Fünf Jahrzehnte nach Einführung der transvenösen Herzstimulation erscheinen z. B. Ergebnisse zu Reizschwellen und Dislokationsraten von Elektroden für einen nationalen wissenschaftlichen Kongress von - vorsichtig formuliert - untergeordneter Bedeutung. Dies hat aber zur Folge, dass auf größeren Kongressen nur die spektakulären Beobachtungen, z. B. hohe Raten an ICDElektrodendefekten, präsentiert werden und diese dann vielfach, sodass ein sehr verzerrtes Bild der klinischen Ergebnisse rhythmologischer Tätigkeit entsteht. 
Die Zusammenlegung von Herbsttagung und Jahrestagung der AG Rhythmologie hat dazu geführt, dass vielfach nur noch erstere wahrgenommen wird, eine der initialen Befürchtungen und Argument gegen eine Zusammenlegung. Dies ist leider soweit fortgeschritten, dass sogar die Hersteller rhythmologischer Medizinprodukte, die die Jahrestagung der Arbeitsgruppen Herzschrittmacher und Arrhythmien mit initiiert und sich im BV-Med zusammengeschlossen haben, die finanzielle Unterstützung der „eigenen“ Jahrestagung weitgehend eingestellt haben. Dies ist im Land der mit Abstand größten Zahl elektrophysiologischer Eingriffe in Europa, von der Schrittmacherimplantation bis zur Pulmonalvenenisolation, nicht gut. Gerade in Deutschland muss klar gemacht werden, dass die hohe Zahl der Eingriffe auch zu einem Vorteil für die Patienten führt. Auch dafür ist es wichtig, die deutsche RhythmologieJahrestagung als Forum zur Präsentation der klinischen Ergebnisse der rhythmologischen Arbeit in Deutschland zu haben. Hier werden vielfach Themen behandelt, für die auf der Frühjahrstagung nicht der richtige Platz ist. In dieser Situation ist es erfreulich, dass die deutsche Rhythmologie-Jahrestagung seitens des Vorstands und Präsidenten der DGK volle Unterstützung erfährt.

Die wichtigste Erkenntnis der diesjährigen Jahrestagung der Arbeitsgruppe Rhythmologie ist jedoch die große Zahl an eingereichten und akzeptierten Abstracts mit einem sehr hohen Anteil an Beiträgen von exzellenter Qualität: Es konnten 119 Abstracts zur Präsentation angenommen werden - das beste Ergebnis seit Kombination von Herbsttagung der DGK und Jahrestagung der AG Rhythmologie und eine Steigerung gegenüber dem Vorjahr um $75 \%$ (• Abb. 1). Dies belegt ein Interesse an rhythmologischer Wissenschaft in Deutschland und eine hohe Qualität der Arbeit in deutschen rhythmologischen Zentren. Diese wissenschaftliche Aktivität ist das stärkste Argument für eine nationale, wissenschaftlich orientierte Rhythmologie-Tagung mit freien Präsentationen. Vielleicht wird es in Zukunft neben Postern auch Vorträge geben. Da die aktive Präsentation eigener Ergebnisse für eine wissenschaftliche Laufbahn von eminenter Bedeutung ist, wünsche ich den Präsentierenden, dass die deutsche Rhythmologie-Jahrestagung ihnen eine Hilfe für die weitere nationale und internationale wissenschaftliche Laufbahn und auch tägliche klinische Tätigkeit sein kann. Nur eine wissenschaftliche Reflektion der eigenen Tätigkeit, verbunden mit dem Mut, Schlussfolgerungen zu ziehen und der Kreativität, Änderungen der eigenen Tätigkeit durchzuführen, kann zu einer Optimierung der eigenen Tätigkeit und Praxis führen.

Interessant ist die Entwicklung der Themen der aktuellen Abstracts der Rhythmologie-Jahrestagung: Fast die Hälfte aller Abstracts befasst sich mit Vorhofflimmern, am häufigsten (65\%) mit der Pulmonalvenenisolation. Folgerichtig haben 5 der 13 Postersitzungen der Rhythmologie-Jahrestagung Vorhofflimmern zum Thema (• Abb. 2). Auch die Tatsache, dass sich 6 der 9 ausgewählten Beiträge für den Hugo-von-ZiemssenPosterpreis mit der Behandlung von Vorhofflimmern befassen, zeigt die Prädominanz dieses Themas. Als Besonderheit ist die Übertragung der Poster auf eine Leinwand und eine Präsentation mit Mikrofon zu nennen, was den Zugang zu einem größeren Publikum ermöglicht. Diese Präsentationen sind als „Spezielle PosterSession" gekennzeichnet. In diesem Jahr wurden hierzu die 3 Sitzungen zu neuen Techniken und Ergebnissen der Pulmonalvenenisolation ausgewählt. Erfreulich sind auch die neuen Themen, die bei der Jahrestagung vorgestellt werden, wie $\mathrm{z}$. B. Laserballon, Contact Force, LAA-Okkluder, subkutaner Defibrillator, „wearable“ Defibrillator, schlafbezogene Atemstörungen und kardiale Kontraktilitätsmodulation.

Besonders freue ich mich, dass es möglich war, den Abstracts der Rhythmologie-Jahrestagung mit dem hier vorliegenden Sonderband der Zeitschrift Herzschrittmachertherapie + Elektrophysiologie $(H+E)$ eine besondere Verbreitung zu ermöglichen und eine physikalisch fassbare, nicht nur virtuelle Präsenz zu vermitteln. Die Zeitschrift $H+E$, heute offizielles Organ der AG Rhythmologie und nationales Organ der EHRA, hatte bis 2003 die Abstracts der Jahrestagung Rhythmologie veröffentlicht, in den letzten Jahren sogar als 2-seitige Kurzartikel. Ich glaube, dass die zusätzliche Veröffentlichung als gedrucktes Abstract-Supplement ein Zeichen setzt, dass die wissenschaftliche Arbeit der Mitglieder unserer Arbeitsgruppe wahrgenommen, gelesen und diskutiert wird. In diesem Zusammenhang gilt der Dank allen Sponsoren, die diesen Sonderband möglich gemacht haben.

Ich wünsche dieser Tagung wie auch der Zukunft der Arbeitsgruppe 1 - Rhythmologie der DGK, auf Dauer eingerichtet und mit dem Recht auf eine eigene Jahrestagung ausgestattet, alles Gute. Auf dass jeder Teilnehmer das Gefühl nachempfinden kann, wie viel Spaß es macht, die eigene Arbeit auszuwerten und die Ergebnisse im erweiterten Kreis unserer Arbeitsgruppe vorzustellen.

Bielefeld, 17.8.2013

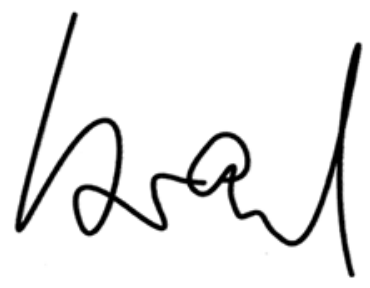

Carsten W. Israel

Tagungspräsident der Jahrestagung der Arbeitsgruppe Rhythmologie

Sprecher der Arbeitsgruppe Rhythmologie

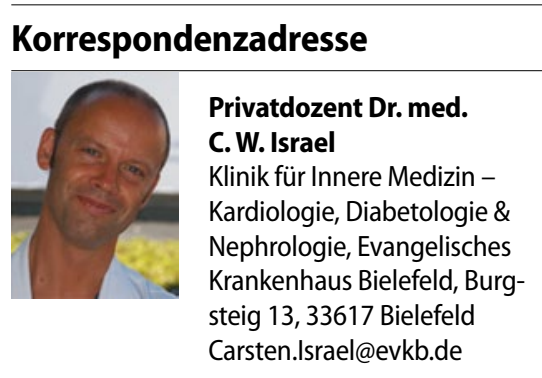

Interessenkonflikt. Der korrespondierende Autor gibt an, dass kein Interessenkonflikt besteht. 